\title{
Estudio fitoquímico y evaluación de la capacidad antioxidante de hojas de Senna reticulata obtenidas en la región andina colombiana
}

\author{
Phytochemical study and evaluation of antioxidant capacity of leaves of Senna \\ reticulata obtained in the Colombian Andean region
}

\section{Estudo fitoquímico e avaliação da capacidade folha antioxidante Senna reticulata obtido na região Andina da Colômbia}

\author{
Javier Andrés Matulevich-Peláez ${ }^{1}$ \\ William Fernando Castrillón-Cardona ${ }^{2}$ \\ Luis Carlos Chitiva-Chitiva ${ }^{3}$
}

Fecha de recepción: septiembre 2016

Fecha de aceptación: abril 2017

Para citar este artículo: Matulevich-Peláez, J.A, Castrillón-Cardona, W. F., y Chitiva-Chivita, L.C. (2017). Estudio fitoquímico y evaluación de la capacidad antioxidante de hojas de Senna reticulata obtenidas en la región andina colombiana. Revista Científica, 29 (2), 149-163. Doi: 10.14483/udistrital.jour.RC.2016.29.a4

\section{Resumen}

El presente trabajo pretende contribuir al estudio fitoquímico de las hojas de esta especie y evaluar su capacidad antioxidante. Para tal fin, a partir del material vegetal colectado en el municipio de Honda (Tolima) e identificado en el Herbario Nacional Colombiano bajo el número de colección COL 573012, se obtuvo el extracto etanólico (131.18 g). Con este, y a través de separaciones por cromatografías en columna (CC), cromatografía en capa delgada (CCD) y cromatografía en capa delgada preparativa (CCDP), se obtuvo una mezcla conformada por eritritol, acetato de isoamilo y ácido L-piroglutámico y el aislamiento de quercetina, hiperósido y camferol. Estos últimos reportados por primera vez para esta especie en nuestro país. La elucidación estructural de los compuestos aislados se llevó a cabo mediante las técnicas de cromatografía de gases acoplada a espectrometría de masas (CG-EM) y resonancia magnética nuclear de carbono
(RMN 13C). La capacidad antioxidante fue evaluada por el método de DPPH, donde se estableció que el extracto etanólico, las fracciones de diclorometano y acetato de etilo y el compuesto identificado como camferol, mostraron un porcentaje de inhibición superior al $40 \%$ tomando como referencia la captación antioxidante del ácido gálico del radical libre DPPH. Palabras Clave: camferol, DPPH, fitoquímica, plantas medicinales.

\begin{abstract}
The purpose of this study was to contribute to the phytochemical study of its leaves and assess to evaluate its antioxidant capacity. For this purpose, from plant material collected in the municipality of Honda (Tolima) and identified in the Colombian National Herbarium collection under the number $\mathrm{COL}$ 573012, the ethanol extract (131.18 g) was obtained from which a mixture consisting of erythritol, isoamyl
\end{abstract}

Universidad Distrital Francisco José de Caldas. Bogotá, Colombia. Contacto: jamatulevichp@udistrital.edu.co Universidad Distrital Francisco José de Caldas. Bogotá, Colombia. Contacto: wfcastrillon@udistrital.edu.co

Universidad Distrital Francisco José de Caldas. Bogotá, Colombia. Contacto: Icchitivac@correo.udistrital.edu.co 
acetate and L-pyroglutamic acid was obtained and an isolation of quercetin, kaempferol and hyperoside, through separations by column chromatographies (CC), thin layer chromatography (TLC) and thin layer preparative chromatography (TLPC). Quercetin, kaempferol and hyperoside were, for this species first reported in our country. The structural elucidation of the isolated compounds was carried out using techniques of gas chromatography-mass spectrometry (GC-MS) and nuclear magnetic resonance of carbon (13C NMR). The antioxidant capacity was evaluated by the method of DPPH, where it was established that the ethanolic extract, fractions of dichloromethane and ethyl acetate and the compound identified as kaempferol, exhibited a percentage of inhibition higher than $40 \%$, taking the antioxidant capture of the free radical DPPH gallic acid as reference. Palavras-chave: DPPH, Kaempferol, phytochemistry, medicinal plants.

\section{Resumo}

O proposito deste trabalho foi fazer a contribuição do estudo fitoquímico das folhas da espécie e avaliar sua capacidade antioxidante. A partir do material vegetal coletado no município de Honda (Tolima), identificado no Herbário Nacional Colombiano com numero de coleção COL 573012, foi possível obter o extrato etánolico (131.18 g); o qual mediante cromatografia em coluna (CC), cromatografia em camada delgada (CCD) e cromatografia em camada delgada preparativa (CCDP) forneceu uma mistura de eritritol, acetato de isoamilo, e acido L-piroglutamico, além do isolamento de quercetína, hiperosido e camferol, estes últimos reportados pela primeira vez para esta espécie no país. A elucidação estrutural dos compostos isolados foi possível mediante as técnicas de cromatografia de gases acoplado com espectrometria de massas (CG-EM) e ressonância magnética nuclear de carbono (RMN 13C). A capacidade antioxidante foi avaliada mediante o método de DPPH, onde foi evidenciado que o extrato etanolico, as frações de diclorometano e acetato de etíla e o composto denominado como camferol, apresentaram um teor de inibição superior ao $40 \%$ tendo em conta como referencia a captação antioxidante do acido do radical livre DPPH.

Palavras chave: DPPH, kaempferol, fitoquímica, plantas medicinais.

\section{Introducción}

La familia Fabaceae es uno de los mayores grupos de plantas con flores (angiospermas) en el mundo, presenta una gran distribución y está muy bien representada en la flora colombiana. En el consenso actual entre especialistas de las Fabáceas, se suele considerarlas como una sola familia Fabaceae, la cual comprende 727 géneros y 19000 especies (Lewis, Schire y Mackinden, 2005). Actualmente en Colombia se registran alrededor de 139 géneros y 1850 especies (Sousa, 2004). México es considerado uno de los principales centros secundarios de diversificación de las Fabaceae, además, este grupo es el segundo más diverso en este país (Sousa, 2004; Judd, Campbell, Kellogg, Stevens y Donoghue, 2002; Forero y Romero, 2005).

En Colombia la especie Senna reticulata se encuentra distribuida en todo el país; la mayor concentración está en los departamentos de Tolima y Cundinamarca, y en menor concentración se encuentra en los departamentos del Amazonas y Arauca (Prance, 1993). Comúnmente, en el departamento del Tolima, esta especie es conocida como montedioco y es utilizada como medicina alternativa en el tratamiento de diferentes infecciones, como purgante, diurético, laxante y abortivo. A nivel mundial es utilizada como insecticida, repelente así como ayuda en el tratamiento del reumatismo, entre otros (Drury y Allen, 1873).

Esta especie es un arbusto leñoso de flores amarillas, pionera en sitios húmedos del Amazonas. Los tallos crecen rápidamente y son de color blan$\mathrm{co}$, produce flores todo el año, incluido el periodo de heladas (invierno), crece en sitios abiertos, pero es capaz de tolerar la sombra y es una planta que resiste a la sequía. Se encuentra en climas templados y algunas veces en páramo, puede alcanzar su crecimiento a una altura de $12 \mathrm{~m}$ alrededor de los 1000 hasta los 2500 msnm. Las hojas de los árboles maduros están caracterizadas por ser compuestas por 8-14 pares de folíolos que son obovadas-oblongas, obtusas, mucronate y glabras en ambos lados (Polhill, Raven y Stirton, 1981; Lavin, 
Brummer, Quire y Maxwell, 1990; Strasburger, 2004).

Los estudios químicos realizados en las hojas y tallo de la especie Senna reticulata han reportado la presencia de flavonoides, alcaloides, taninos, antraquinonas, saponinas, esteroides, terpenos y glicósidos, de los cuales se destacan los compuestos crisofanol, fisciona, emodina, 1,3,8-trihidroxiantraquinona, 1,6,8-trihidroxi-3-metoxiantraquinona, camferol, $\beta$-sistosterol, estigmasterol, $\alpha$-amirina y $\beta$-amirina (Dos Santos, Goretti y Filho, 2008; Vargas, 2007).

En cuanto a los estudios de actividad biológica para hojas, corteza y flores de esta especie se ha reportado la evaluación de diferentes actividades como: antioxidante y citotóxica para fracciones en medio acuoso y de etanol (Cárdenas y Pérez, 2013; Lizcano, Bakkali y Ruiz, 2010; González et al., 2014), antimicrobiana e inmunológica para fracciones de hexano (De Oliveira, 2009), antiinflamatoria y cicatrizante para fracciones en metanol (Vargas, 2007), antiparasitaria y antibacteriana (Nunes, 2007), citotóxica para fracciones en etanol (Camacho Phillipson, Croft, Marshall y Ghazanfar, 2003) y antiglucemiante para fracciones en medio acuoso (Isaza, Cristancho, Cruz y Castrillón., 2006).

El objetivo principal de este estudio, teniendo en cuenta el bajo volumen de información que se encuentra de esta especie en nuestro país, es contribuir al estudio fitoquímico de hojas de Senna reticulata y evaluar su capacidad antioxidante por el método de DPPH.

\section{Materiales y métodos}

\section{Recolección del material vegetal}

La especie vegetal fue recolectada en el municipio de Honda, Tolima ( $5^{\circ} 11^{\prime} 28^{\prime \prime} \mathrm{N}, 74^{\circ} 44^{\prime} 34^{\prime \prime} \mathrm{O}$ ). Se recolectaron $700 \mathrm{~g}$ de hojas, las cuales fueron secadas a temperatura ambiente y reducidas de tamaño en un molino de grano 1c100 doméstico para su posterior extracción; una muestra testigo fue enviada al Herbario Nacional de Colombia para su determinación taxonómica, la cual fue clasificada como Senna reticulata bajo el código n. ${ }^{\circ}$ COL 573012, identificada por el biólogo Carlos Alberto Parra.

\section{Obtención de extractos y fracciones}

El extracto etanólico de hojas de Senna reticulata (E.EtOH.Sr.H) se obtuvo a partir de $550 \mathrm{~g}$ de material vegetal seco y molido empleando etanol al 96 \% por el método de maceración en frio. El extracto obtenido fue floculado con agua en relación 1.5:1 $\left(\mathrm{H}_{2} \mathrm{O}\right.$ : E.EtOH.Sr.H) con el fin de eliminar interferencias e impurezas, posteriormente filtrado y concentrado a presión reducida a $40^{\circ} \mathrm{C}$. Además, le fueron realizadas pruebas químicas de precipitación y/o coloración con el fin de conocer los posibles grupos de metabolitos secundarios presentes en él (Sanabria, 1999).

Para el fraccionamiento del extracto se empleó el método de extracción líquido-líquido continuo con solventes de polaridad creciente, obteniéndose las fracciones de hexano (Fx.Hex.Sr.H), diclorometano (Fx.DCM.SrH) y acetato de etilo (Fx. AcOEt.Sr.H). Cada una de estas fracciones fue sometida a separaciones continuas a través de CC, CCD y CCDP para finalmente aislar una mezcla de tres componentes y tres flavonoides.

\section{Técnicas para la elucidación estructural y caracte- rización de los compuestos aislados}

Para determinar la mezcla y los compuestos obtenidos se utilizó un cromatógrafo de gases con detector selectivo de masas Shimadzu QP2010 plus, ubicado en el laboratorio de química de la Universidad Distrital Francisco José de Caldas. La separación se realizó en una columna capilar SHRXi-5MS de $30 \mathrm{~m}$ de longitud $\times 0.25 \mathrm{~mm} \times 0.25$ $\mu \mathrm{m}$ con una inyección en modo Split (10:1); el gas de arrastre utilizado fue helio (grado 5.0) con flujo constante de $1.2 \mathrm{~mL} / \mathrm{min}$. La programación de la temperatura del horno fue de $50{ }^{\circ} \mathrm{C}(2 \mathrm{~min})$ a $15^{\circ} \mathrm{C} /$ 
min hasta $200^{\circ} \mathrm{C}(2 \mathrm{~min})$ a $10^{\circ} \mathrm{C} / \mathrm{min}$ hasta $300^{\circ} \mathrm{C}$ (10min) para un tiempo total de análisis de $34 \mathrm{mi}-$ nutos; la temperatura de la línea de transferencia fue de $275^{\circ} \mathrm{C}$ y de la cámara de ionización de $230^{\circ} \mathrm{C}$. Los espectros de masas de los compuestos aislados fueron tomados en un equipo Shimadzu MS QP2010 dotado con una sonda de inserción directa y analizador de masas cuadrupolar. Utilizando un modo de ionización electrónica (IE) a 70 eV y una temperatura de la cámara de ionización de $230^{\circ} \mathrm{C}$.

Los espectros de RMN ${ }^{13} \mathrm{C}$ fueron tomados en un espectrómetro Bruker Avance del Laboratorio de Resonancia Magnética Nuclear del Departamento de Química de la Pontificia Universidad Javeriana. Los análisis se realizaron a $75 \mathrm{MHz}$ para ${ }^{13} \mathrm{C}$, empleando solventes deuterados (acetona-d6 y metanol-d4), con tetrametilsilano (TMS) como referencia interna.

\section{Evaluación de la capacidad antioxidante}

La capacidad antioxidante se determinó mediante el método de DPPH al extracto etanólico (E.EtOH. Sr.H), las fracciones de diclorometano (Fx.DCM. Sr.H) y acetato de etilo (Fx.AcOEt.Sr.H) y al compuesto identificado como camferol. Los análisis fueron realizados en el laboratorio del Grupo de Investigación en Farmacología Vegetal y Terapéutica Alternativa de la Fundación Universitaria Juan N. Corpas.

A partir de un stock de 500 ppm de ácido gálico se realizaron ocho diluciones en serie $(500,250$, $125,62.5,31.2,15.6,7.8$ y $3.9 \mathrm{mg} / \mathrm{L})$ y se midieron las absorbancias a $520 \mathrm{~nm}$ en un equipo Accu Reader $^{2000}$; este mismo tratamiento se llevó a cabo con las muestras ensayadas. Para el tratamiento y validación del método analítico de dilución utilizado se realizó una estandarización del patrón de referencia utilizando ácido gálico, validando el método mediante una curva de calibración con un coeficiente de regresión lineal de 0.9997, utilizando concentraciones de 1.041, 2.041, 3.041, 4.041 y $5.041 \mu \mathrm{m} / \mathrm{mL}$.
De acuerdo a las mediciones, se estableció la función matemática que relaciona las variables del porcentaje de inhibición y concentración con un coeficiente de correlación de 0.990232. El cálculo de los porcentajes de inhibición (\%l) y la IC50 se evaluaron midiendo las absorbancias de las muestras en tiempos parciales de 0 y 30 minutos hasta reacción total a los 60 minutos, donde se determinó como punto final del ensayo la disminución de la absorbancia en la muestra de estudio y en el cambio de color de cada una de las soluciones. Se utilizó un control positivo (ácido gálico $+\mathrm{MeOH}$ ) y uno negativo (DPPH $+\mathrm{MeOH}$ ) con el fin de descartar posibles errores en el método a la hora de la determinación de las absorbancias para las muestras ensayadas. La trazabilidad de cada una de las soluciones preparadas se realizó mediante el patrón de referencia el ácido gálico.

El análisis estadístico para la cuantificación de la capacidad antioxidante (\% inhibición y IC50) se realizó utilizando el programa Minitab 15, de acuerdo a los reportes establecidos por triplicado, donde se establecieron coeficientes de correlación, análisis de varianza, correlación de variables, determinación de mínimos cuadrados y regresión lineal para el extracto, las fracciones y compuestos con promisoria capacidad.

\section{Resultados y discusión}

\section{Marcha fitoquímica preliminar}

Los resultados de la marcha fitoquímica preliminar permitieron establecer la presencia de flavonoides, quinonas, taninos, alcaloides, terpenos y glucósidos cardiotónicos. Por el contrario, no presenta cumarinas, saponinas y carotenoides, lo cual coincide con los reportes químicos de algunas de las especies de la familia Fabaceae como la Bauhinia variegata L., Senna alata, y Senna spectabilis, entre otras. Para el género Senna se han reportado una gran variedad de compuestos como esteroides, terpenoides y flavonoides (Vargas, 2007), lo que ha permitido clasificar a la especie como promisoria 
de sustancias bioactivas de diversa naturaleza química e importantes para la farmacognosia.

\section{Metabolitos secundarios aislados}

La purificación por CC y CCD del extracto etanólico y las fracciones de hexano, diclorometano y acetato de etilo condujeron al aislamiento e identificación de una mezcla conformada por eritritol, acetato de isoamilo y ácido L-piroglutámico (mezcla SrH1), dos flavonoides: camferol (compuesto $\mathrm{SrH} 2$ ) y quercetina (compuesto $\mathrm{SrH} 4$ ) y un flavonoide glucosilado identificado como hiperósido (compuesto $\mathrm{SrH} 3$ ). Los compuestos $\mathrm{SrH} 2, \mathrm{SrH} 3$ y $\mathrm{SrH} 4$ fueron elucidados por métodos espectroscópicos y por comparación con los datos descritos en la literatura. La mezcla SrH1 fue analizada por CG-EM.

\section{Composición de la mezcla SrH1}

La corriente iónica total (TIC) del análisis por CGEM para la mezcla $\mathrm{SrH1}$ arrojó tres señales con tiempos de retención de 8.372, 9.584 y 12.998 minutos. La comparación de los espectros de masas de las respectivas señales con los de la librería NIST08 indica que corresponden a eritritol, acetato de isoamilo y ácido L-piroglutámico con porcentajes de coincidencia de 93, 95 y $97 \%$, respectivamente. El eritritol ha sido reportado para la familia Fabaceae, específicamente en la especie Erythina variegata (Rahman Sultana, Faruquee y Ferdous, 2007; Schnakenberg Stutte, Guo y Clark, 1990) y se reporta por primera vez en la especie Senna reticulata. El acetato de isoamilo ha sido reportado ampliamente para diversas familias vegetales como: Cucurbitaceae (González, Hernández, González, Bustos y Rosales, 2009), Lamiaceae (González, González, Palomares y Tábata, 2010), Verbenaceae (Nuñez Aguado, Bela, Vonka y Sansberro, 2007), Musaceae (Pontes Pereira y Camara, 2012), Ceratocystidaceae (Christen, 1995) y Rubiaceae (Guyot Cros y Vincent, 1982); para la familia Fabaceae se reporta por primera vez al igual que el ácido L-piroglutámico.

\section{Elucidación estructural del compuesto $\mathrm{SrH} 2$}

El compuesto $\mathrm{SrH} 2(86.3 \mathrm{mg})$ se obtuvo como un sólido de color amarillo, insoluble en hexano, poco soluble en acetona y soluble en metanol. La respectiva comparación de los espectros de masas con la librería NIST 08 permitió identificar el compuesto como 3,5,7-trihidroxi-2-(4-hidroxifenil)-4H-1-benzopiran-4-ona también conocido como camferol con un $98 \%$ de coincidencia. El espectro de masas muestra el ion molecular con una señal muy intensa a m/z 286, asignada también como el pico base y además las señales en $\mathrm{m} / \mathrm{z} 287,285,259,258,257,231,229,213,165$, 153, 136, 134 y 121 que de acuerdo a la literatura (Raymond Xiu-Sheng y Chris, 2004) corresponden al compuesto camferol.

$\mathrm{El}$ análisis por $\mathrm{RMN}{ }^{13} \mathrm{C}$ confirmó la estructura del compuesto identificado como camferol. El espectro muestra diversas señales las cuales son atribuidas a 15 carbonos. Se observan señales características de carbonos aromáticos y olefínicos en $\sigma 145.9,135.3,175.9,97.9,164.1,93.3$, 156.8, 102.6, 121.6, 129.4, 115.0, 159.2, 115.0 y $131.7 \mathrm{ppm}$. Además se observa una señal en la región de campo bajo del espectro a $\sigma 208.3$ ppm correspondiente a un carbono carbonilico. Luego del análisis de los datos de $\mathrm{RMN}{ }^{13} \mathrm{C}$, EM y de la comparación con datos de la literatura (Xiao Wu, Shi, Hang y Aisa , 2006), se asignaron los desplazamientos químicos para cada uno de los carbonos de la molécula la cual se identificó como camferol.

El camferol ha sido reportado previamente para la especie Senna reticulata en un estudio desarroIlado en Perú (Vargas, 2007) y por primera vez en especies colombianas en este estudio. También ha sido identificado en otras especies de la familia Fabaceae, como en Bauhinia manca (Achenbach, Stocker y Constela, 1988) y descrito ampliamente para las familias vegetales: Nictaginaceae (Stintzing et al., 1998), Clusiaceae (Nguemeving et al., 2006), Brassicaceae (Ning et al., 2007), PhyIlanthaceae (Gutiérrez, 2012; Sousa et al., 2007; 
Calixto Adair, Niraldo, Valdir y Rosendo, 1998) y Asclepiadaceae (Sikorska y Matlawska, 2001).

\section{Elucidación estructural del compuesto SrH3}

El compuesto $\mathrm{SrH} 3$ (88.5 mg) se obtuvo como un sólido de color amarillo, insoluble en hexano y soluble en acetona. La respectiva comparación de los espectros de masas con la librería NIST08 permitió identificar la presencia del compuesto Quercetina-3 $\beta$-D-galactopiranósido, también conocido como hiperósido, con un porcentaje de coincidencia de $97 \%$. El espectro de masas mostró una señal en $\mathrm{m} / \mathrm{z} 302$, la cual fue asignada como el pico base, y además las señales en $\mathrm{m} / \mathrm{z}$ 286, 285, 229, $228,165,153$ y 150 , que de acuerdo con la literatura (Jing Dawei, Jianming y Shu, 2013), corresponde al compuesto hiperósido.

El compuesto $\mathrm{SrH} 3$ (hiperósido) ha sido ampliamente reportado para la familia Rosaceae (Xun et al., 2010; Olszewska, 2005) y descrito en las familias vegetales: Hipericaceae (Raghu Mahal, Srinivasan, Jagani y Vijayan, 2015), Grossulariaceae (Dajun et al., 2010), Asclepiadaceae (Sikorska y Matlawska, 2001) y reportado por primera vez en la especie Senna reticulata.

\section{Elucidación estructural del compuesto SrH4}

El compuesto SrH4 (37.8 mg) se obtuvo como un sólido de color amarillo, insoluble en hexano y soluble en acetona. La respectiva comparación de los espectros de masas con la librería NIST08 permitió identificar el compuesto como 2-(3,4-dihidroxifenil)-3,5,7-trihidroxi-4H-4-cromona también conocido como Quercetina con un porcentaje de coincidencia del $98 \%$. El espectro de masas muestra el ion molecular con una señal muy intensa a m/z 302, asignada también al pico base y además las señales en $\mathrm{m} / \mathrm{z} 303,301,286$, 258, 257, 231, 229, 213, 165, 153, 137, 121 y 109 que según Skliar y Toso (2002) ,y a estudios realizados por Matulevich (2013), corresponden al compuesto Quercetina.
El análisis por $\mathrm{RMN}{ }^{13} \mathrm{C}$ confirmó la estructura del compuesto identificado como Quercetina. El espectro muestra diversas señales, las cuales son atribuidas a 15 carbonos. Se observan señales de carbonos aromáticos y olefínicos en $\sigma$ 148.7, 138.3, 172.5, 158.9, 96.4, 162.9, 91.5, 103.8, 127.9, 112.9, 142.7, 148.7, 112.9, 127.9 ppm, además se observa una señal a campo bajo del espectro en $\sigma 208.3$ ppm asignada al carbono carbonilico. Luego del análisis de los datos de RMN ${ }^{13} \mathrm{C}$, EM y de la comparación con datos de la literatura (Fathiazad Delazar, Amiri y Sarker, 2006), se asignaron los desplazamientos químicos para cada uno de los carbonos de la molécula la cual se identificó como Quercetina.

El compuesto SrH4 (Quercetina) ha sido ampliamente reportado y aislado para la familia Phyllanthus en especies tales como: Phyllanthus Orbularissi HBK (Gutiérrez, 2012), P. Caroliniensis (Cechinel et al., 1996), P. muellerianus (Chen y Ren, 1997), P. urinaria (Xu, Zha, Qin, Zhang y Yang, 2007) y descritas ampliamente para las familias vegetales: Asclepiadaceae (Sikorska y Matlawska, 2001), Brassicaceae (Ning et al., 2007), Laminaceae (Ling Robbins y Harnly, 2007; Molina, 2011), Nyctaginaceae (Stintzing et al., 1998), Myrtaceae (Méndez, 2006; Guzmán y Henríquez, 2007), Ericaceae (Harborne y Williams, 1973; Matulevich, 2013), reportado también en la familia Fabaceae por la especie Bauhinia Microstachya (Vargas, 2007) y reportado por primera vez en la especie Senna reticulata.

\section{Evaluación de la capacidad antioxidante}

En cuanto a las propiedades biológicas de los compuestos encontrados se han reportado estudios de la evaluación de diferentes actividades como: antioxidante para fracciones en medio acuoso y de etanol (Lizcano Bakkali y Ruiz, 2010; Cárdenas y Pérez, 2013; González et al., 2014), antimicrobiana e inmunológica para fracciones de hexano (De Oliveira, 2009), antiinflamatoria y cicatrizante para fracciones en metanol (Vargas, 2007), 
citotóxica para fracciones en etanol (Camacho et al., 2003) y antiglucemiante para fracciones en medio acuoso (Isaza et al., 2006).

De acuerdo a esto, un estudio realizado a Hypericum coadunatum, una especie productora de numerosas sustancias polifenólicas, entre ellos los flavonoides como la quercetina y derivados acetilados, muestran que las actividades bilógicas estudiadas presentan un efecto positivo frente a sustancias antitumorales, antiinflamatorias, antivíricas y antibacteriales. Se afirma que dichas propiedades están dadas por la capacidad antioxidante de estas sustancias (Flores, 2016).

Otro estudio realizado a la especie Lentinula edodes reporta una gran actividad antioxidante debido a la presencia de flavonoides de tipo glicosidado, donde los resultados ponen de manifiesto que el extracto crudo es el que presenta mayor actividad antioxidante (41.11-92.9\%) seguido del extracto en acetato de etilo $\left(\mathrm{E}_{\mathrm{AcOE}}\right)(2.3-76.5 \%)$, el extracto en cloroformo $\left(\mathrm{E}_{\mathrm{CHCl} 3}\right)(27.9-75.4 \%)$ y el extracto en hexano $\left(\mathrm{E}_{\mathrm{H}}\right)(5.0-37.0 \%)$. La caracterización parcial permitió inferir que la mayor actividad antioxidante del extracto crudo es atribuida a la presencia de compuestos polifenólicos (polifenoles y flavonoides), determinados por primera vez para esta especie (Castañeda y Ramírez, 2009).

La determinación de la capacidad antioxidante del extracto, las fracciones y el compuesto identificado como camferol se determinó mediante el método de DPPH, donde los tratamientos que presentaron un porcentaje de inhibición superior al $40 \%$ (tabla 1) fueron aceptados para el método utilizado, de acuerdo a la comparación con el patrón de referencia y curva de calibración para este caso el ácido gálico (figura 1).

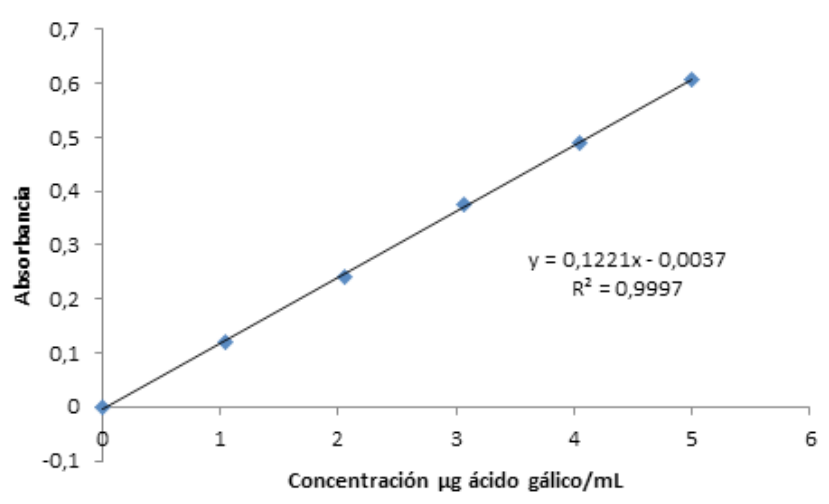

Figura 1. Curva de calibración para el porcentaje de inhibición.

Tabla 1. Porcentaje de inhibición e IC50 de DPPH de tratamientos ensayados de hojas de Senna reticulata.

\begin{tabular}{|c|c|c|c|c|c|c|}
\hline \multirow{2}{*}{ Muestras } & \multicolumn{5}{|c|}{ \% Inhibición DPPH } & \multirow{2}{*}{$\begin{array}{c}\mathrm{IC}_{50}{ }^{*} \\
\operatorname{VCEAG}(\mathrm{ppm})\end{array}$} \\
\hline & 550 ppm & $250 \mathrm{ppm}$ & 125 ppm & 62.5 ppm & $31.2 \mathrm{ppm}$ & \\
\hline E.EtOH.Sr.H & $\begin{array}{c}92.29 \pm \\
0.092\end{array}$ & $\begin{array}{c}88.97 \pm \\
0.091\end{array}$ & $\begin{array}{c}63.65 \pm \\
0.082\end{array}$ & $\begin{array}{c}56.73 \pm \\
0.074\end{array}$ & $\begin{array}{c}54.96 \pm \\
0.075\end{array}$ & 96.1 \\
\hline Fx.DCM.Sr.H & $\begin{array}{c}79.60 \pm \\
0.093\end{array}$ & $\begin{array}{c}68.70 \pm \\
0.093\end{array}$ & $\begin{array}{c}38.82 \pm \\
0.075\end{array}$ & $(-)$ & $(-)$ & 183.8 \\
\hline Fx.AcOEt.Sr.H & $\begin{array}{c}77.65 \pm \\
0.101\end{array}$ & $\begin{array}{c}54.25 \pm \\
0.092\end{array}$ & $(-)$ & $(-)$ & $(-)$ & 265.1 \\
\hline Camferol & $\begin{array}{c}85.37 \pm \\
0.100\end{array}$ & $\begin{array}{c}79.52 \pm \\
0.090\end{array}$ & $\begin{array}{c}73.40 \pm \\
0.085\end{array}$ & $\begin{array}{c}62.23 \pm \\
0.090\end{array}$ & $\begin{array}{c}36.61 \pm \\
0.069\end{array}$ & 130.0 \\
\hline
\end{tabular}

*VCEAG: capacidad antioxidante equivalente al ácido gálico. (-) \% de inhibición inferior al 30 \%. 
De acuerdo a los resultados presentados en la tabla 1, el extracto etanólico (E.EtOH.Sr.H), las fracciones diclorometano y acetato de etilo (Fx. DCM.Sr.H y Fx.AcOEt.Sr.H) y el compuesto identificado como camferol presentaron un porcentaje de inhibición superior al $40 \%$, mostrando para cada caso una efectiva captación del radical libre DPPH. Además, se observó que a medida que la concentración de cada uno de los tratamientos ensayados disminuye el porcentaje de inhibición también lo hace de forma proporcional. Las concentraciones que superaron el $40 \%$ de inhibición se encontraban dentro del rango de (500 a 125 ppm) presentando estas la mayor capacidad antioxidante mostrándose un error estándar estimado de \pm 0.090 para el ensayo de inhibición de DPPH en cada una de las muestras realizadas.

Para la interpretación de los valores obtenidos durante el ensayo DPPH se estimaron los valores estadísticos correspondientes para el control positivo de observación en cada ensayo efectuado para el ácido gálico. Los datos estadísticos se resumen en las tablas 2 y 3 .

Tabla 2. Coeficientes de correlación.

\begin{tabular}{lllll}
\hline & $\begin{array}{l}\text { Mínimos } \\
\text { cuadrados }\end{array}$ & Estándar & Estadístico & \\
Predictor & Estimado & Error & $\mathrm{T}$ & Valor-P \\
Intercepto & 34.5236 & 0.7214 & 47.86 & 0.012 \\
Pendiente & 0.087160 & 0.003534 & 24.66 & \\
\hline
\end{tabular}

Tabla 3. Análisis de varianza.

\begin{tabular}{llllll}
\hline Fuente & GL & SC & MC & Razón-F & Valor-P \\
Regresión & 1 & 1590.2 & 1590.2 & 608.27 & 0.045 \\
Error residual & 6 & 15.7 & 2.6 & & \\
$\begin{array}{l}\text { Total } \\
\text { correlación }\end{array}$ & 7 & 1605.9 & & & \\
\hline
\end{tabular}

*GL: grados de libertad, SC: suma de cuadrados, MC: cuadrado medio.
De acuerdo al análisis estadístico realizado, el valor-P es menor a 0.05 , indicando que existe una relación estadísticamente significativa entre el porcentaje de inhibición y la concentración con un nivel de confianza del $95.0 \%$, el valor estadístico R-cuadrada indica que el modelo ajustado explica $98.9 \%$ de la variabilidad en \% de inhibición de las muestras analizadas, con un coeficiente de correlación igual a 0.990232, indicando una relación significativa entre cada una de las variables. Los valores estadísticos indican que el modelo es adecuado para la interpolación de los valores obtenidos para el extracto, fracciones y compuestos obtenidos.

La determinación de la actividad antioxidante del extracto, las fracciones y compuesto se consideró como activo y válido aquellos que presentaron un porcentaje de inhibición superior al $40 \%$, valor utilizado como referencia de acuerdo al equivalente a la mitad de la actividad presentada por el ácido gálico como patrón control. Dichos porcentajes corresponden al IC50, estableciéndose una relación lineal positiva entre \% vs. IC50. De acuerdo a las cuatro muestras mediante el método DPPH, todas presentaron actividad antioxidante promisoria.

El estadístico R-cuadrada indica el modelo ajustado y explica en $60.5 \%$ la poca variabilidad en el porcentaje de inhibición, ajustándose a un error estándar estimado de 8.689 y un coeficiente de correlación 0.813409, donde la linealidad del modelo es aceptable para el ensayo, pero únicamente para las concentraciones de 550 ppm, 250 ppm y 125 ppm mostrando linealidad (figura 2). Para el extracto E.EtOH.SrH se calculó el porcentaje de inhibición acorde a los valores indicados en la tabla 1, y la IC50 mediante el análisis estadístico de supervivencia y confiabilidad probit al $50 \%$ de los datos obtenidos, determinando un $92.296 \%$ para 550 ppm, 88.971\% para 250 ppm, $63.652 \%$ para 125 ppm, $56.737 \%$ para 62.5 ppm y $54.964 \%$ para 31.2 ppm con un IC50 de 96.1321 ppm (VCEAG). Los datos estadísticos y los valores calculados se discriminan en la tabla 4. 


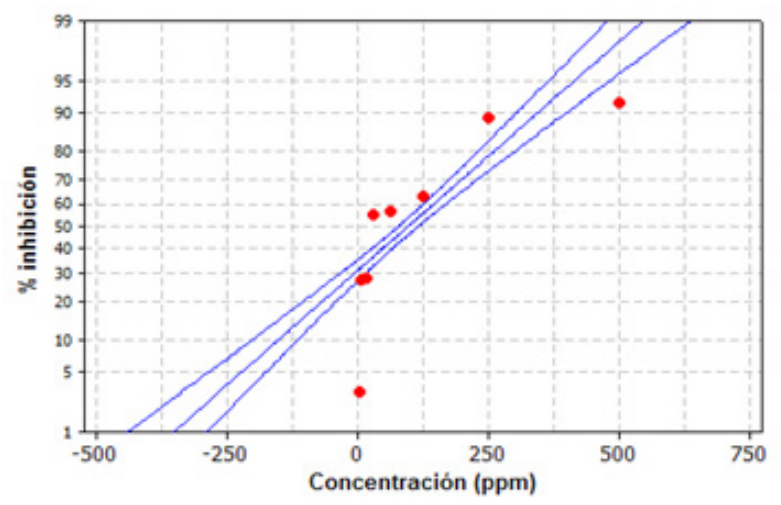

Figura 2. Probabilidad del porcentaje de inhibición para el extracto E.EtOH.Sr.H * \% AA $=33.6+0.146$ * concentración. Coeficiente de relación: 0.813409. Líneas azules: límite inferior, linealidad del modelo y límite superior. Puntos rojos: concentración 3.9, 7.8, 15.6, 31.2, 62.5, 125, 250 y 500 ppm.

Tabla 4. Estadística para el extracto E.EtOH.Sr.H.

\begin{tabular}{lllll}
\hline & \multicolumn{1}{c}{ Mínimos cuadrados } & \multicolumn{1}{c}{ Estándar } & \multicolumn{1}{c}{ Estadístico } & \\
Predictor & Estimado & Error & $\mathrm{T}$ & Valor-P \\
Intercepto & 33.598 & 8.689 & 3.87 & 0.008 \\
Pendiente & 0.14580 & 0.04257 & 3.45 & 0.014 \\
\hline
\end{tabular}

El estadístico R-cuadrada indica el modelo ajustado y explica en $88.5 \%$ la posible variabilidad en el porcentaje de inhibición, ajustado a un error estándar de 3.162, y un coeficiente de correlación de 0.949635 donde la linealidad del modelo es aceptable para el ensayo (figura. 3). Para la fracción Fx.DCM.Sr.H se calculó el porcentaje de inhibición acorde a los valores indicados en la tabla 1, y la IC50 mediante el análisis estadístico de supervivencia y confiabilidad probit al $50 \%$ de los datos obtenidos, determinando un $79.609 \%$ para 550 ppm, $68.705 \%$ para 250 ppm y $38.829 \%$ para 125 ppm con un IC50 de 183.839 ppm (VCEAG). Los datos estadísticos y los valores calculados se discriminan en la tabla 5.

El estadístico R-cuadrada indica el modelo ajustado y explica en 93.9 \% la posible variabilidad en el porcentaje de inhibición, ajustado a un error estándar de 2.626, y un coeficiente de correlación de 0.973716 donde la linealidad del modelo es aceptable para el ensayo (figura 4). Para la fracción Fx.AcOEt.Sr.H se calculó el porcentaje de inhibición acorde a los valores indicados en la tabla 1, y la IC50 mediante el análisis estadístico de supervivencia y confiabilidad probit al $50 \%$ de los datos obtenidos, determinando un $77.659 \%$ para 550 ppm y $54.255 \%$ para 250 ppm con un IC50 de 265,104 ppm (VCEAG). Los datos estadísticos y los valores calculados se discriminan en la tabla 6 .

El estadístico R-cuadrada indica el modelo ajustado y explica en $55.2 \%$ la posible variabilidad en el porcentaje de inhibición, ajustado a un error estándar de 9.413, y un coeficiente de correlación de 0.784953 donde la linealidad del modelo es poco aceptable para el ensayo, pero sí muestra linealidad únicamente para las concentraciones de 550, 250, $125,62.5$ y 31.2 (figura 5). Para el compuesto $\mathrm{SrH} 2$ se calculó el porcentaje de inhibición acorde a los valores indicados en la tabla 1, y la IC50 mediante el análisis estadístico de supervivencia y confiabilidad probit al $50 \%$ de los datos obtenidos, determinando un $85.372 \%$ para 550 ppm, 79.521 para 250 ppm, $73.404 \%$ para 125 ppm, $62.234 \%$ para 62.5 ppm y $36.613 \%$ para 31.2 ppm, con un IC50 de 130.050 ppm (VCEAG). Los datos estadísticos y los valores calculados se discriminan en la tabla 7. 


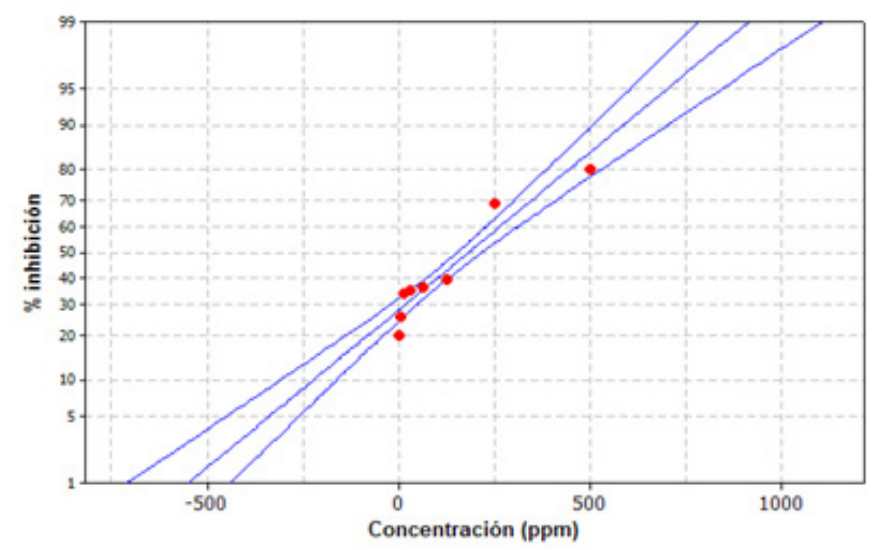

Figura 3. Probabilidad del porcentaje de inhibición para la fracción Fx.DCM.Sr.H * \% AA = 28,1 + 0,115* concentración. Coeficiente de relación: 0.949635. Líneas azules: Límite inferior; linealidad del modelo y límite superior. Puntos rojos: concentración 3.9; 7.8; 15.6; 31.2; 62.5; 125; 250 y 500 ppm.

Tabla 5. Estadística para la fracción Fx.DCM.Sr.H.

\begin{tabular}{lllll}
\hline & Mínimos cuadrados & Estándar & Estadístico & \\
Predictor & Estimado & Error & T & Valor-P \\
Intercepto & 28.057 & 3.162 & 8.87 & 0.000 \\
Pendiente & 0.11500 & 0.01549 & 7.42 & 0.000 \\
\hline
\end{tabular}

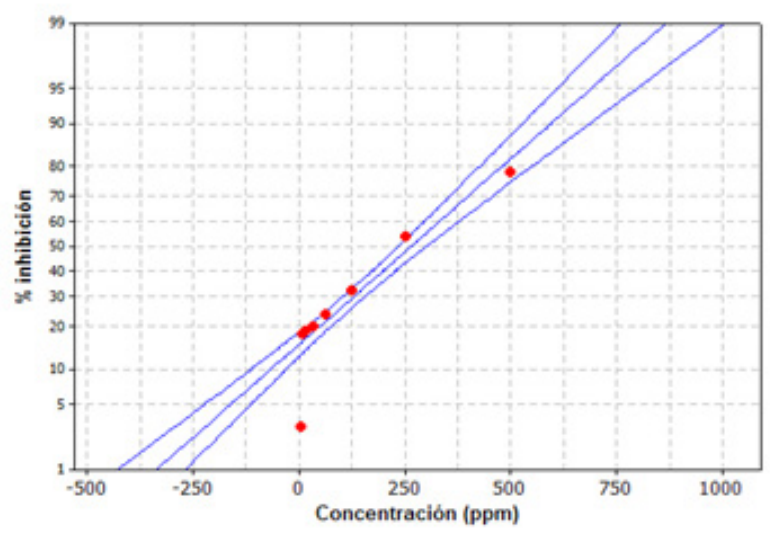

Figura 4. Probabilidad del porcentaje de inhibición para la fracción Fx.AcOEt.Sr.H. * \% AA = 14,2 + 0,135 * concentración. Coeficiente de relación: 0.973716. Líneas azules: Límite inferior; linealidad del modelo y límite superior. Puntos rojos: concentración 3.9; 7.8; 15.6; 31.2; 62.5; 125; 250 y 500 ppm.

Tabla 6. Estadística para la fracción Fx.AcOEt.Sr.H.

\begin{tabular}{lllll}
\hline & Mínimos cuadrados & Estándar & Estadístico & \\
Predictor & Estimado & Error & $\mathrm{T}$ & Valor-P \\
Intercepto & 14.229 & 2.626 & 5.42 & 0.002 \\
Pendiente & 0.13470 & 0.01286 & 10.47 & 0.000 \\
\hline
\end{tabular}




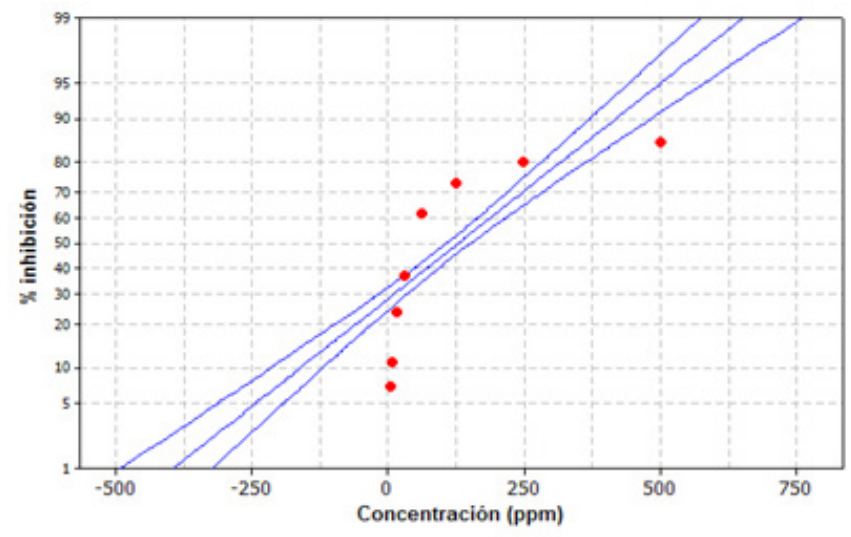

Figura 5. Probabilidad del porcentaje de inhibición para el compuesto $\mathrm{SrH} 2 .{ }^{*} \% \mathrm{AA}=29.6+0.143 *$ concentración. Coeficiente de relación: 0.784953. Líneas azules: límite inferior, linealidad del modelo y límite superior. Puntos rojos: concentración 3.9, 7.8, 15.6, 31.2, 62.5, 125, 250 y 500 ppm

Tabla 7. Estadística para el compuesto $\mathrm{SrH} 2$.

\begin{tabular}{lllll}
\hline & Mínimos cuadrados & Estándar & Estadístico & \\
Predictor & Estimado & Error & T & Valor-P \\
Intercepto & 29.558 & 9.413 & 3.14 & 0.020 \\
Pendiente & 0.14311 & 0.04611 & 3.10 & 0.021 \\
\hline
\end{tabular}

Cada uno de los modelos lineales analizados evaluó el rango de concentraciones preparadas para el ensayo y determinó la sensibilidad, linealidad y reproducibilidad del método. El rango de concentraciones evaluado fue de 3.9 a 500 ppm, con coeficientes de correlación de 0.9496-0.9737 para las fracciones Fx.DCM.Sr.H y Fx.AcOEt.Sr.H, presentando una linealidad óptima para los ensayos. Para el extracto E.EtOH.Sr.H y el compuesto $\mathrm{SrH} 2$ con coeficientes de correlación 0.813409 y 0.784953 no evidenciaron linealidad respecto al análisis de los datos; debido a que el \% de inhibición de la actividad antioxidante se generó a concentraciones altas únicamente (500, 250 y 125 ppm). De acuerdo a esto, y los errores de absolutos presentados, el ensayo de captación del radical libre DPPH funciona adecuadamente dentro del rango de concentraciones descritas, pero puede presentar interferencias a la hora de variar las concentraciones del ensayo.
De acuerdo a los resultados obtenidos, el extracto (E.EtOH.Sr.H), las fracciones (Fx.DCM.Sr.H y Fx.AcOEt.Sr.H) y el compuesto $\mathrm{SrH} 2$ presentaron un \% de inhibición adecuado para el ensayo realizado, mostrando para cada uno una efectiva captación del radical libre DPPH. A medida que la concentración disminuía por cada ensayo, el \% de inhibición disminuía; las concentraciones que superaron el $40 \%$ de inhibición estaban dentro del rango de (500 a 125 ppm), presentando promisoria actividad antioxidante para cada una de las muestras en cada ensayo determinado.

Otros estudios realizados para la familia Fabaceae reportan una fuerte capacidad de eliminación contra el radical libre DPPH, como es el caso del extracto metanólico obtenido a partir de semiIlas de Ramorinoa girolae Speg, donde se atribuye esta capacidad principalmente a los compuestos fenólicos y flavonoides presentes (Luna et al., 2013). Para las especies Binatus lathyrus, Trifolium 
pannonicum y Anthyllis aurea se ha reportado una fuerte capacidad antioxidante tomando como referencia los valores del porcentaje de inhibición del antioxidante equivalente al Trolox (Teac) (Godevac et al, 2008). Para el extracto en EtOH de Bauhinia kalbreyeri se evidenció un alto potencial antioxidante superior al $90 \%$ al inhibir la propagación de radicales libre utilizando DPPH, donde la corteza resultó más activa que la hoja para el ensayo realizado. Al comparar estos resultados con los reportados en este estudio se observa que el extracto etanólico de Senna reticulata sobrepasa la capacidad antioxidante de Bauhinia kalbreyeri ya que a una concentración de 550 ppm se obtuvo un porcentaje de inhibición del $92.29 \%$.

Para el género Senna en particular se han reportado estudios de su capacidad antioxidante, como es el caso de los carotenoides presentes en flores de Senna multiglandulosa, donde se obtuvieron porcentajes de inhibición superiores al $42.65 \%$ (Cornejo, 2011). De acuerdo con lo anterior, se puede establecer qué especies vegetales pertenecientes a la familia Fabaceae y específicamente al género Senna se pueden clasificar como fuertes agentes antioxidantes gracias a su riqueza metabólica, lo cual las convierte en posibles alternativas terapéuticas frente al tratamiento del estrés oxidativo.

\section{Conclusiones}

El trabajo fitoquímico desarrollado para las hojas de la especie vegetal Senna reticulata permitió el aislamiento e identificación de una mezcla de compuestos conformada por eritritol, acetato de isoamilo y ácido L-piroglutámico; dos compuestos de tipo flavonoide identificados como quercetina y camferol y un flavonoide glucosilado identificado como hiperósido.

La evaluación de la actividad antioxidante permitió establecer que la presencia de flavonoides y compuestos fenólicos presentes en la especie, son los responsables de la capacidad antioxidante presentada en cada ensayo para el extracto E.EtOH. Sr.H, las fracciones Fx.DCM.Sr.H y Fx.AcOEt.Sr.H, y el compuesto $\mathrm{SrH} 2$; donde todas captaron significativamente con un \% de inhibición superior al $40 \%$ tomando como referencia la captación antioxidante del ácido gálico en el radical libre DPPH. Esto hace parte, de un aporte importante a la industria farmacéutica de los agentes antioxidantes en el tratamiento de diferentes enfermedades. Permitiendo así, un aporte al conocimiento químico y biológico de la familia Fabaceae en Colombia, mediante el estudio fitoquímico de sus metabolitos fijos y la determinación de su capacidad antioxidante de los extractos, fracciones y metabolitos secundarios mayoritarios presentes en las hojas de Senna reticulata.

\section{Referencias}

Achenbach, H., Stocker, M. y Constela, M. (1988). Flavonoides y otros constituyentes de la Bauhinia Manca. Fitoquímica, 27(6), 1835-1841.

Calixto, J., Adair, R., Niraldo, P., Valdir, C. y Rosendo, A. (1998). A review of the plants of the genus Phyllanthus: Their chemistry, pharmacology and therapeutic potential. Med. Res. Rev., 18(4), 225-258.

Castañeda, A. y Ramírez, J. (2009) Estudio de los compuestos polifenólicos, con énfasis en flavonoides, del hongo Lentinula edodes y determinación de la actividad antioxidante (tesis de maestría en Química), pp. 9-87. Facultad de Ciencias, Departamento de Química, Universidad Nacional de Colombia, Bogotá.

Cárdenas, A. y Pérez, J. (2013). Especies vegetales investigadas por sus propiedades antimicrobianas, inmunomoduladoras e hipoglucemiantes en el departamento de Caldas (Colombia-Sudamérica). Revista Biosalud Manizales, 12(1), 59-82.

Camacho, R., Phillipson, D., Croft, L., Marshall, J. y Ghazanfar, A. (2003). Ensayos de extractos de plantas para actividades antiprotozoarios y citotóxicos. Elsevier Journal of Ethnopharmacology, 89(2-3), 185-191.

Christen, P. (1995). Producción de aromas por fermentación en medio sólido. Tópicos de investigación y posgrado, 4(2), 102-109. 
Cechinel, V., Santos, A., De Campos, R., Obdulio, G., Yunes, G., Ferrari, F. y Calixto, J. (1996). Chemical and pharmacological studies of PhyIlanthus caroliniensis in mice. Journal of Pharmacy and Pharmacology, 48(12), 1231-1236.

Cornejo, A. (2011). Extracción, identificación, cuantificación y determinación de la actividad antioxidante de los carotenoides presentes en las flores de Senna multiglandulosa a través de cuatro métodos de extracción (trabajo de grado). Ingeniería en Biotecnología, Departamento de Ciencias de la vida, Universidad de las Fuerzas Armadas, Sangolquí, cuador.

Chen, T. y Ren, L. (1997). Studies on the anticancer constituents of matsumura leaf flower (Phyllanthus matsumurae). I. Isolation and identification of flavonoid compounds. Institute Clinical Medicine, Sino Japan Friendship Hospital, Beijing, Peop. Rep. China., 28(1), 5-7.

Dos Santos, R., Goretti, M. y Filho, R. (2008). Constituintes químicos do caule de Senna reticulata Willd. (Leguminoseae). Revista Química Nova, 31(8), 1979-1981.

De Oliveira, A. (2009). Actividad antimicrobiana e inmunológica in vitro de dos extractos de Senna reticulata (Willd.) Irwin y Barneby (matapasto) y Vismia guianensis (Aubl.) (lacre) (tesis de maestría). Ciencias Farmacéuticas, Universidade Estadual Paulista, Campus de Araraquara.

Dajun, H., Huang, Y., Ayupbek, A., Dongyo, G., Yang, Y., Akber, H. e Ito, Y. (2010). Separation and Purification of Flavonoids from Black $\mathrm{Cu}$ rrant Leaves by High-Speed Countercurrent Chromatography and Preparative HPLC. National Library of Medicine, 33(5), 615-628.

Drury H., y Allen, H. (1873). Las plantas útiles de la India. Cassia Alata. P. 119-120.

Fathiazad, F., Delazar, A., Amiri, R. y Sarker, S. (2006). Extraction of flavonoids and quantification of rutin from waste Tabacco leaves. Iranian Journal of Pharmaceutical Research, 3, 222-227.

Forero, E. y Romero, C. (2005). Estudios en leguminosas colombianas. Bogotá: Editora Guadalupe LTDA.
Flores, F. D. (2016). Obtención de flavonoles de plantas superiores. Actividad biológica (trabajo de grado), p. 4-28. Facultad de Ciencias, Universidad de la Laguna, Tenerife, España.

Godevac, D., Zdunic, G., Savikin, K., Vajs, V. y Menkovic, N. (2008). La actividad antioxidante de nueve especies Fabaceae crecimiento en Serbia y Montenegro. ELSEVIER Fitoterapia., 79(3), 185-187.

González, M., Serrano, E., Murillo, R., Mora, S., Araya, V., Ramírez, W. y Hoyos, M. (2014). Estudio de cuatro plantas con uso medicinal tradicional cultivadas en las regiones Huetar Norte y Atlántica de Costa Rica. Tecnología en marcha, 27(4), 69-77.

González, S., Hernández, A., González, M., Bustos, H. y Rosales, T. (2009). Comparación de dos métodos de extracción de compuestos volátiles en sandía (Citrullus lanatus T.). Quehacer científico, 1(7), 23-27.

González, J., González, H., Palomares, R. y Tábata, A. (2010). Microextracción en fase sólida de compuestos volátiles en albahaca (Ocimum basilicum L.). Acta universitaria, 21(1), 17-22.

Guyot, B., Cros, E. y Vincent, J. (1982). Caractérisation et identification des composés de la fraction volatile d'un café vert Arabica sain et d'un café vert arabica puant. Biblioteca conmemorativa Orton, 26(4), 279-289.

Gutiérrez, Y. (2012). Estudio farmacognóstico de Phyllanthus orbicularis HBK, especie endémica de Cuba. La Habana: Editorial Universitaria.

Guzmán, M. y Henríquez, A. (2007). Determinación de la actividad antimicrobiana de las fracciones obtenidas de la cromatografía de columna (n-hexano: acetato de etilo $70 \%$, acetato de etilo puro y metanol puro) procedentes del extracto diclorometánico de la goma-resina de Eucalyptus citriodo (trabajo de grado). Para optar el título de Licenciado en Química y Farmacia, Universidad de el Salvador, Facultad de Química Y Farmacia. P, 1-124.

Harborne, J. y Williams, C. (1973). A chemotaxonomic survey of flavonoids and simple phenols 
in leaves of the Ericaceae. Botanical J. Linn., 66, 37-54.

Isaza, G., Cristancho, L., Cruz, A. y Castrillón, H. (2006). Efectos de la Senna reticulata en la glicemia de ratones normoglicémicos e hiperglicémicos. Revista Biosalud, 5, 61-67.

Judd, W., Campbell, E., Kellogg, P., Stevens, M. y Donoghue, J. (2002). Plantas sistemáticas: un enfoque filogenético (2da ed.). EE.UU.: Sinauer Associates Inc.

Jing, Y., Dawei, Q., Jianming, G. y Shu, J. (2013). La identificación de los principales metabolitos de hiperosido producidas por las bacterias intestinales humanas utilizando la espectrometría de masas de cromatografía líquida de ultra-cuadrupolo tiempo de vuelo. Journal of Ethnopharmacology, 147(1), 174-179.

Lavin, M., Brummer, R., Quire, B. y Maxwell, L. (1990). Análisis filogenético de una comunidad de la biodiversidad vegetal en el oeste de América del Norte. Nueva York: Garden Botanical.

Lewis, G., Schire, B. y Mackinden, B. (2005). Tribú Caesalpiniae. Avances en la sistemática de Leguminosas (Fabaceae). Buenos Aires: Botanic Gardens.

Ling, L., Robbins, R. y Harnly, J. (2007). Identification and quantification of flavonoids of Mexican oregano (Lippia graveolens) by LC-DAD-ES/MS analysis. Food Comp. Anal., 20, 361-369.

Lizcano, L., Bakkali, F. y Ruiz, M. (2010). La actividad antioxidante y el contenido de polifenoles de los extractos acuosos de plantas amazónicas colombianas con uso medicinal. Revista química de los alimentos, 119, 1566-1570.

Luna, L., Pigni, N., Claveira, T., Monferran, M., Magdalena, Maestri, D. y Tapia, A. (2013). Ramorinoa girolae Speg (Fabaceae) semillas, una especie argentina de comida tradicional indígena: la composición de nutrientes y actividad antioxidante. Elsevier, 31(1), 120-128.

Matulevich, J. (2013). Estudio fitoquímico de hojas, flores y frutos de Bejaria resinosa Mutis ex Linné filius (Ericaceae) y evaluación de su actividad antiinflamatoria (trabajo de grado).
Departamento de Química, Facultad de Ciencias, Pontificia Universidad Javeriana, Bogotá, Colombia.

Méndez, R. (2006). Determinación de la actividad antibacteriana del extracto diclorometánico de la goma-resina de Eucalyptus citriodora (Eucalipto) (trabajo de grado). Facultad de Química y Farmacia, Universidad del Salvador, El Salvador.

Molina, M. (2011). Determinación de antioxidantes fenólicos en plantas aromáticas del género Lippia Sp. (familia Verbenaceae) empleando cromatografía de gases y cromatografía líquida de alta eficiencia (trabajo de grado). Postgrado en Química, Facultad de Ciencias, Universidad Industrial de Santander, Bucaramanga, Colombia.

Nguemeving, J., Azebaze, A., Kuete, V., Carly, N., Beng, P., Meyer, M. y Nkengfack, A. (2006). Laurentixanthones $A$ and $B$, antimicrobial xanthones from Vismia laurentii. Phytochemistry, 67(13), 1341-1346.

Ning, S., Wei, X., Hongfeng, G., Xiaoqiu, L., Yibo, W. y Xiaoling, N. (2007). Several flavonoids from Capsella bursa-pastoris (L.) Medic. Several flavonoids from Capsella bursa-pastoris (L.) Medic. Asian Journal of Traditional Medicines, 2(5).

Nunes, S. (2007). Estudio químico y farmacológico de la Senna reticulata Willd (tesis de doctorado). Universidade Federal do Ceará, Fortaleza, Brasil.

Nuñez, M., Aguado, M., Bela, A., Vonka, C. y Sansberro, P. (2007). Farmacognosia y fitoquímica de Lippia turbinata G., Verbenaceae. (Pharmacognosy and phytochemistry of Lippia turbinata G., Verbenaceae). Boletín Latinoamericano y del Caribe de Plantas Medicinales y Aromáticas, 6(5), 262-263.

Olszewska, M. (2005). Flavonoids from prunus serotina ehrh. Department of Pharmacognosy, 62(2), 127-133.

Prance, G. (1993). Notas sobre la vegetación de la Amazonia. III. Terminología de tipos de bosques amazónicos sujetas a la inundación. Revista Brittonia, 31(1), 26-38. 
Polhill, R., Raven, W. y Stirton, C. (1981). Evolución y sistemática de las leguminosas. Avances sistemáticos en leguminosas. Inglaterra: Royal Botanic Garden.

Pontes, M., Pereira, J. y Camara, J. (2012). Dinámica espacio de cabeza microextracción en fase sólida combinada con unidimensional gas espectrometría de cromatografía-masa como una poderosa herramienta para diferenciar cultivares de banano en base a su perfil de metabolitos volátiles. Elsevier Food chemistry, 134(4), 2509-2520.

Raghu, C., Mahal, H., Srinivasan, R., Jagani, H. y Vijayan, P. (2015). A pulse radiolysis study of hyperoside isolated from Hypericum mysorense. Radiation Physics and Chemistry, 107, 149-159.

Raymond, E., Xiu-Sheng, M. y Chris, D. (2004). A fragmentation study of kaempferol using electrospray quadrupole time-of-flight mass spectrometry at high mass resolution. International Journal of Mass Spectrometry, 231(2), 157-167.

Rahman, M., Sultana, S., Faruquee, C. y Ferdous, F. (2007). Investigaciones fitoquímicas y biológicas de Erythrina variegata. Saudi Pharmaceutical Journal, 15(2).

Sanabria, A. (1999). Colección de especies vegetales y análisis fitoquímico preliminar. Universidad Nacional de Colombia, cap I p. 127-140.

Sousa, M., Ousingsawat, J., Seitz, R., Puntheeranurak, S., Regalado, A., Schmidt, A. y Kunzelmann, K. (2007). An extract from the medicinal plant Phyllanthus acidus and its isolated compounds induce airway chloride secretion: a potential treatment for cystic fibrosis. Molecular Pharmacology, 71(1), 366-376.

Sousa, M. (2004). El género Inga (Leguminosae: Mimosoideae) del sur de México y Centroamérica, estudio previo para la flora de la Amazonia Peruana. Jardín Botánico Missouri, 80(1), 223-269.
Sikorska, M. y Matlawska, I. (2001). Kaempferol, isorhamnetin and their glycosides in the flowers of Asclepias syriaca L. Polish Pharmaceutical Society, 58(4), 269-272.

Skliar, M., y Toso, R. (2002). Aislamiento, identificación y cuantificación de compuestos con actividad gastroprotectora presentes en Centaurea solstitialis. Ciencia veterinaria, Facultad de ciencias veterinarias, Universidad Nacional de la Pampa, Argentina.

Stintzing, F., Kammerer, D., Schieber, A., Adama, H., Nacoulma, O. y Carle, R. (1998). Betacyanins and phenolic compounds from Amaranthusspinosus L. and Boerhaviaerecta L. Phytochemistry, 14(2A), 501-507.

Strasburger, E. (2004). Tratado de botánica. Barcelona: Omega.

Schnakenberg, C., Stutte, C., Guo, C. y Clark, T. (1990). Concentraciones de poliol en la soja (Glycine max L.) influenciado por el estrés y bioregulación. Advanced Society of America. 4(6), 73-87.

Vargas, C. (2007). Estudio de la actividad cicatrizante y antiinflamatoria del extracto alcohólico de las hojas de la Senna reticulata (Willd.) H. Irwin y Barneby (Retama) (tesis de maestría). Universidad Nacional Mayor de San Marcos, Lima, Perú.

Xiao, P., Wu, H., Shi, H., Hang, B. y Aisa, H. (2006). Kaempferol and quercetin flavonoids from Rosa rugosa. Chemistry of Natural Compounds, 42(6), 736-737.

Xu, M., Zha, Z., Qin, X., Zhang, X. y Yang, C. (2007). Phenolic antioxidants from the whole plant of Phyllanthus urinaria. Chemistry \& Biodiversity, 4(9), 2246-2252.

Xun, L., Dong, W., Si-Yuan, W., Xian-Sheng, M., Wen-Jie, Z., Xi-Xiang, Y. y Ting-Guo, K. (2010). LC Determination and Pharmacokinetic Study of Hyperoside in Rat Plasma after Intravenous Administration. The Pharmaceutical Society of Japan, 130(6), 873-879. 